\title{
Effect of level of confusability on reporting letters from briefly presented visual displays
}

\author{
CAROL L. KRUMHANSL and EWART A. C. THOMAS \\ Stanford University, Stanford, California 94305
}

\begin{abstract}
In a report paradigm, two letters are presented on a trial which are either confusable (e.g., $P$ and $R$ ) or nonconfusable (e.g., $P$ and $M$ ) in terms of visual features. Across trials, interletter distance, retinal location, duration, and visual field are varied. Identification accuracy on confusable trials was generally lower than on nonconfusable trials, and this effect of level of confusability increased with distance from the fixation point, decreased with duration, and was smaller on the central letter than on the more peripheral letter. A quantitative model, incorporating aspects of the interactive channels model (Estes, 1972) and feature perturbation model (Wolford, 1975), is developed and tested. One parameter of the model measures the effective similarity between two letters after lateral inhibition has occurred, and other parameters measure the probability of feature perturbation in foveal and peripheral directions.
\end{abstract}

Studies investigating the effect of level of confusability in detection paradigms have consistently found that performance is worse when the noise characters are confusable with the target character than when the noise and target characters are dissimilar (Estes, 1972; Gardner, 1973; McIntyre, Fox, \& Neale, 1970; Shiffrin \& Gardner, 1972). Other variables which have been found to influence performance in identifying letters are serial position, retinal location, and interletter letter distance. U-shaped serial position curves have typically been generated when performance is plotted as a function of distance from a fixation point, and the shape of these curves has been found to be largely independent of stimulus duration (Estes, Allmeyer, and Reder, 1976; Taylor and Brown, 1972; Townsend, Taylor, and Brown, 1971). A number of studies have suggested that, under the stimulus conditions (size, luminance, retinal locus) normally employed in letter-perception experiments, retinal location may not be a strong determinant of performance (Bryden, 1966; Crovitz \& Schiffman, 1965). However, retinal location has been found to interact with serial position in a number of studies (Bouma, 1970; Estes et al., 1976; Krumhansl \& Thomas, 1976; Wolford \& Hollingsworth, 1974b). Finally, letter identification is generally facilitated by increasing interletter distance (Eriksen \& Eriksen, 1974; Estes

The work was supported by a National Institutes of Health training grant (MH-10478-09) to the first author, a National Science Foundation grant (GB-43275) to the second author, and a National Institute of Mental Health grant (MH-21747) to Richard C. Atkinson, whose assistance is gratefully acknowledged. The helpful comments of Nancy Cantor and two reviewers are also greatly appreciated. Reprint requests may be sent to Carol L. Krumhansl, Department of Psychology, Stanford University, Stanford, California 94305.
\& Wolford, 1971; Shaw, 1969; Strangert and Brännström, 1975, Wolford \& Hollingsworth, 1974a), although the opposite effect was found in a study in which nonletter positions were occupied by noise characters (Krumhansl \& Thomas, 1976).

The present study was designed to investigate the effect of level of confusability in a report paradigm and the interaction between this variable and duration, retinal location, string position, and interletter distance. The results will be discussed in terms of two models which have been proposed for the letter-identification process, the interactive channels model (Estes, 1972, 1974) and the feature perturbation model (Wolford, 1975). While the interactive channels model is directly concerned with level of confusability, we try to make more explicit the predictions of this model concerning the interaction between this variable and other display variables. The question of level of confusability is not addressed in the feature perturbation model, but, from the assumptions of the model, various predictions concerning this variable and its interactions can be derived.

Estes $(1972,1974)$ proposed the interactive channels model to account for the results of detection experiments in which performance was found to decrease as the similarity between noise and target characters increased. Although the interactive channels model was proposed to account for performance in detection tasks, it is equally applicable to a report paradigm. In this model, it is assumed that if confusable characters, which share features, are presented close together in the visual field, then competition for access to feature detectors occurs. This competition occurs because excitation of a particular inut channel to a feature 
detector exerts an inhibitory effect on other nearby input channels going to the same feature detector. Performance is poorer with confusable characters than with nonconfusable characters, since, owing to inhibition, there is a certain probability that no input channel is available for transmission to an essential feature detector. It is assumed that the strength of inhibition decreases as intercharacter distance increases, so this model would predict that the effect of level of confusability would decrease as intercharacter distance increases. Finally, since the density of input channels is assumed to decrease with increased distance from the center of the fovea, it would be expected that the effect of level of confusability would be accentuated at peripheral locations. This would be true since, with fewer channels, there would be a greater probability that inhibitory effects from a nearby character would leave no input channel free for transmission to a required feature detector when confusable characters were presented. However, since performance is relatively poor at peripheral locations, the increased effect of confusability at these locations may not be seen in the detection probability if there are "floor" effects or if confusability has a proportional rather than an additive effect on detection probability.

Wolford (1975) has proposed a feature perturbation model to account for performance in a variety of report experiments. This model assumes that letter features and interletter blanks are extracted from the visual display and that, during processing of information in the sensory store, perturbations occur in the spatial ordering of the extracted features. The probability that a feature (or blank) is extracted is assumed to decrease with increased distance from the center of the fovea. The probability of a feature moving from its correct feature group is also assumed to increase with distance from the center of the fovea such that foveal transpositions are more likely than peripheral transpositions. Perturbations are assumed to occur over time, so that the later a feature appears in the processing order, the greater the probability of perturbation of that feature. Difficulties arise in the identification process when perturbations occur between feature groups, since features may be missing from a feature group or extraneous features (from other letters) may be included in the feature group. In this model, it is assumed that as long as a feature group consists of a subset of the features belonging to exactly one letter, the letter is correctly identified. Otherwise, if an extraneous feature is included in the feature group, or if the feature group is a subset of the features belonging to two or more letters, the letter is not correctly identified.

Although the model as presented in Wolford (1975) is not explicitly concerned with the effects of level of confusability, it is possible to discuss various predictions concerning level of confusability in terms of the model. It might be supposed that perturbations occurring between feature groups would not have as large a detrimental effect when the presented letters share features as when the presented letters do not share features. This would be true because the arrival of a feature into a feature group would not cause difficulty in identification if the feature was compatible with the features already in the feature group. If it is assumed that confusable letters share more features than do nonconfusable letters, then, in general, this model would seem to predict that, in report paradigms, performance should be better when letters are confusable that when letters are nonconfusable. Also, two other predictions concerning level of confusability can be derived from the model. First, since the probability that a feature moves from one feature group to another feature group is assumed to decrease as the distance between the presented letters increases, it would be predicted that the effect of level of confusability would decrease as the interletter distance increased. Second, since it is assumed that features tend to move foveally, it would be expected that the detrimental effect of feature transpositions on identification of a letter at a given location would be larger if a second letter was presented in a peripheral location than if a second letter was presented in a foveal location. ${ }^{1}$ Taken together with the prediction that transpositions have a larger effect on identifying nonconfusable than confusable letters, this predicts that the largest effect of feature transpositions should be found on the more foveal of two letters when they are nonconfusable.

The predictions made by the models of Estes (1972, 1974) and Wolford (1975) concerning the effect of level of confusability are tested in the present study. In this experiment, subjects were asked to report as many letters as possible from a two-letter array. The two letters presented on a trial were chosen so as to be confusable (e.g., $P$ and $R$ ) or nonconfusable (e.g., $P$ and $M$ ) in terms of visual features. The trials were varied with respect to stimulus duration, interletter distance, and retinal location, so that the effect of these variables, as well as relative position (whether central or peripheral), could be evaluated. Of primary interest was the way in which these variables interact with level of confusability, and the overall effect of level of confusability.

\section{METHOD}

\section{Subjects}

The 16 Stanford University students who served as subjects received credit toward introductory psychology. The subjects had normal to corrected-to-normal vision and participated in two test sessions which lasted approximately $45 \mathrm{~min}$ each. 


\section{Apparatus}

The experiment was run using a programmable CRT display (Imlac Corporation PDS-1) interacting with a PDP-10 timesharing computer system. The test display appeared on the screen in green characters against a dark gray background. The screen was viewed from a distance of approximately $50 \mathrm{~cm}$. The subjects typed their responses using the typewriter-like keyboard in front of the CRT screen.

\section{Stimulus Materials}

The premask and postmask arrays consisted of a horizontal string of 10 noise characters (dollar signs) with a small $x$ in the center of the array. The array subtended a horizontal visual angle of $4.00^{\circ}$ and a vertical angle of $.375^{\circ}$ when viewed from a distance of $50 \mathrm{~cm}$. The noise characters and letters measured $.25^{\circ}$ wide and $.375^{\circ}$ high, intercharacter spaces were $.125^{\circ}$, and the center $\times$ measured $.25^{\circ}$ wide and $.25^{\circ}$ high. Fourteen blocks of 32 trials were constructed. On each trial, two letters appeared in the left or the right visual field, such that one letter always appeared in Retinal Location 3 and the second letter appeared in Retinal Location 1, 2, 4, or 5 in the same visual field where retinal location is defined as shown in Table 1. The stimulus remained on the screen for either $65 \mathrm{msec}$ (short duration) or $90 \mathrm{msec}$ (long duration). Confusable trials consisted of two letters both drawn without replacement from one of the three sets [B, D, R, P], $[T, L, F, H]$, or $[M, N, W, V]$. On nonconfusable trials, the two letters were drawn from two different sets so that all letters appeared equally often in nonconfusable and confusable trials. The choice of these sets of confusable letters is based on Townsend's (1971) observation that average intragroup confusability is larger than average intergroup confusability. Table 1 shows a sample of left visual field trials; right visual field trials were mirror images. Thirty-two trial types were defined by visual field (left or right), level of confusability (confusable or nonconfusable), duration (short or long), and position of the distractor (Retinal Location 1, 2,4 , or 5). A trial of each type appeared once in each block of trials, so that across blocks each trial type appeared 14 times.

\section{Procedure}

At the start of the first test session, the subjects were given instructions about their task. They were told that on each trial the following sequence of events would occur: (a) The word "READY" would appear centered on the CRT screen. (b) The subject would then press the spacebar on the typewriter-like keyboard in front of the CRT screen. (c) The premask array would appear centered on the screen. (d) After the subject had fixated on the $x$ in the center of the array, the subject would again press the spacebar. (e) The premask array would disappear, and the two test letters would be displayed briefly. (f) The postmask array would then appear and remain on the screen for $700 \mathrm{msec}$. (g) Finally, the word "RESPONSE" would be written on the screen and the subject would type his response. The subjects were told to respond with as many letters as possible, and were encouraged to guess on the basis of partial information. On those trials on which the subject's response consisted of two letters, they were told to type them in a left-to-right order.

In each test session, 10 practice trials were followed immediately by 7 trial blocks, so that the 14 trial blocks were evenly divided between the two sessions, which were scheduled on different days. The blocks were randomly ordered for each subject, and trials were randomly ordered within the blocks.

\section{RESULTS}

\section{Visual Field, Retinal Location, Relative Position, and Interletter Distance}

Figure 1 shows average performance at each retinal location as a function of interletter distance and relative position, where a letter is defined to be
Table 1

Sample Trial Types

\begin{tabular}{lccccccccccc}
\hline & & & & \multicolumn{1}{c}{ Retinal Location } \\
\cline { 2 - 9 } Display Type & 5 & 4 & 3 & 2 & 1 & X & 1 & 2 & 3 & 4 & 5 \\
\hline Confusable & & & L & & T & X & & & & & \\
Nonconfusable & & & T & B & & X & & & & \\
Confusable & & P & B & & & X & & & & \\
Nonconfusable & R & & H & & & X & & & & \\
Pre-and Postmask & $\$$ & $\$$ & $\$$ & $\$$ & $\$$ & X & & & & & \\
\hline
\end{tabular}

in the central relative position if it is the closer of the two presented letters to the fixation point, and in the peripheral relative position if it is the farther of the two presented letters from the fixation point. Central letters were presented in Retinal Locations 1, 2, and 3 , and peripheral letters were presented in Retinal Locations 3, 4, and 5. On each trial, a letter was presented in Retinal Location 3 in either the left or the right visual field, and this letter will be called a target. A second letter was also presented on each trial in Retinal Location 1, 2, 4, or 5 in the same visual field, and this letter will be called a distractor. Analyses of variance of performance scores collapsed across duration and level of confusability were done separately for targets and distractors, since retinal location was held constant for targets but not for distractors.

For targets, performance was higher if the target, always presented in Retinal Location 3, was peripheral than if it was central relative to the distractor $[F(1,15)=54.37, p<.01]$. The difference between central and peripheral targets was larger in

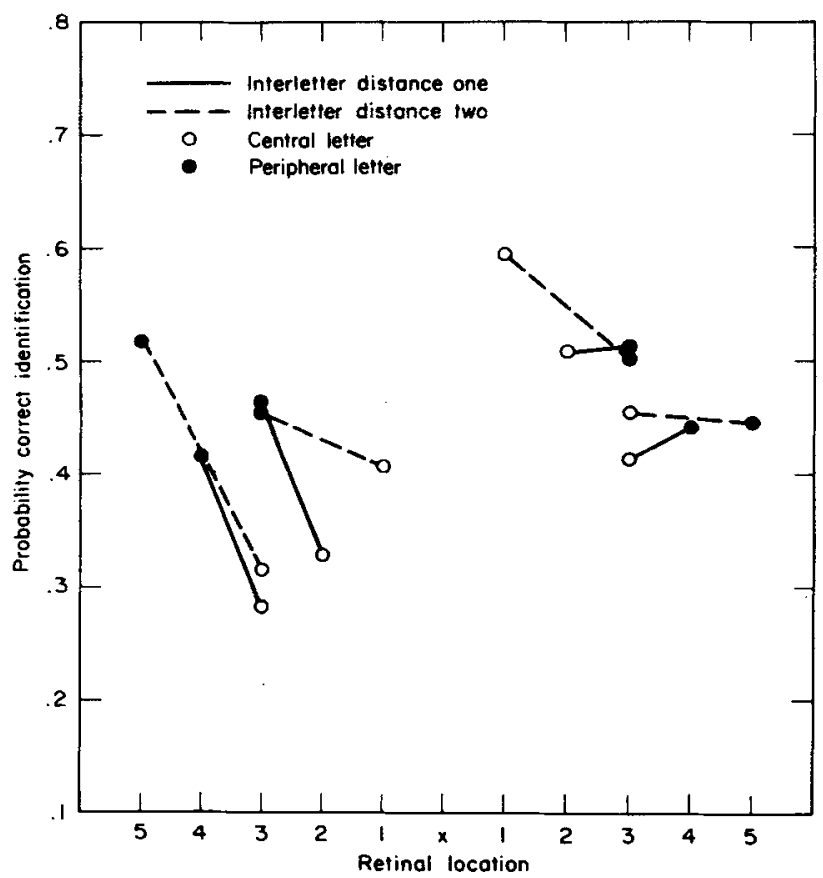

Figure 1. Probability of correct letter identification as a function of retinal location, interletter distance, and relative position in the two visual fields. 
the left visual field than in the right visual field $[F(1,15)=13.15, p<.01]$. No other effects were significant for targets. In particular, identifying the target letter was not significantly affected by the distance between the target and the distractor.

For distractors, there was a main effect of visual field, with performance higher in the right visual field than in the left visual field $[F(1,15)=6.565$, $p<.05]$. In addition, performance was higher for distractors which were peripheral relative to the target in the left visual fielci, while performance was higher for distractors which were central relative to the target in the right visual field $[\mathrm{F}(1,15)=21.456$, $\mathrm{p}<.01]$, indicating a left-to-right bias in letter identification. Also, an effect of interletter distance was found, with performance being poorer at Retinal Locations 2 and 4 than at Retinal Locations 1 and 5 $[\mathrm{F}(1,15)=20.520, \mathrm{p}<.01]$. Finally, there was a significant triple-order interaction between visual field, interletter distance, and serial position $[F(1,15)$ $=6.711, \mathrm{p}<.05]$.

\section{Level of Confusability and Duration}

Figure 2 shows average performance at each retinal location as a function of the duration of presentation and level of confusability. An analysis of variance was done on identification scores collapsed across visual field and including all retinal locations (both targets and distractors). The performance score used for Retinal Location 3 was performance at that retinal location averaged over distractor location. There was a main effect of level of confusability, with performance higher if the letters were nonconfusable than if the letters were confusable $[F(1,15)=17.700, p<.01]$. Performance increased with duration $[F(1,15)=161.348, p<.01]$, and duration interacted with level of confusability $[F(1,15)=4.717, p<.05]$, such that the effect of level of confusability was larger at the shorter duration. An increasing linear trend was found in the difference between performance on nonconfusable and confusable trials as the distance from the fixation point increased $[F(1,60)=6.39, p<.05]$. In addition, the effect of retinal location was significant $[F(4,60)=5.799, p<.01]$, although the linear trend was not significant. The interaction between duration and retinal location was significant $[F(4,60)$ $=2.999, \mathrm{p}<.05]$, as was the triple-order interaction between level of confusability, duration, and retinal location $[F(4,60)=2.759, p<.05]$.

Table 2 shows the difference between the nonconfusable and confusable conditions as a function of relative position and interletter distance. There was a tendency for the difference to be smaller for central letters than for peripheral letters (the difference was on the average .021 and .056 for central and peripheral letters, respectively). While relative position is confounded with retinal location, a direct

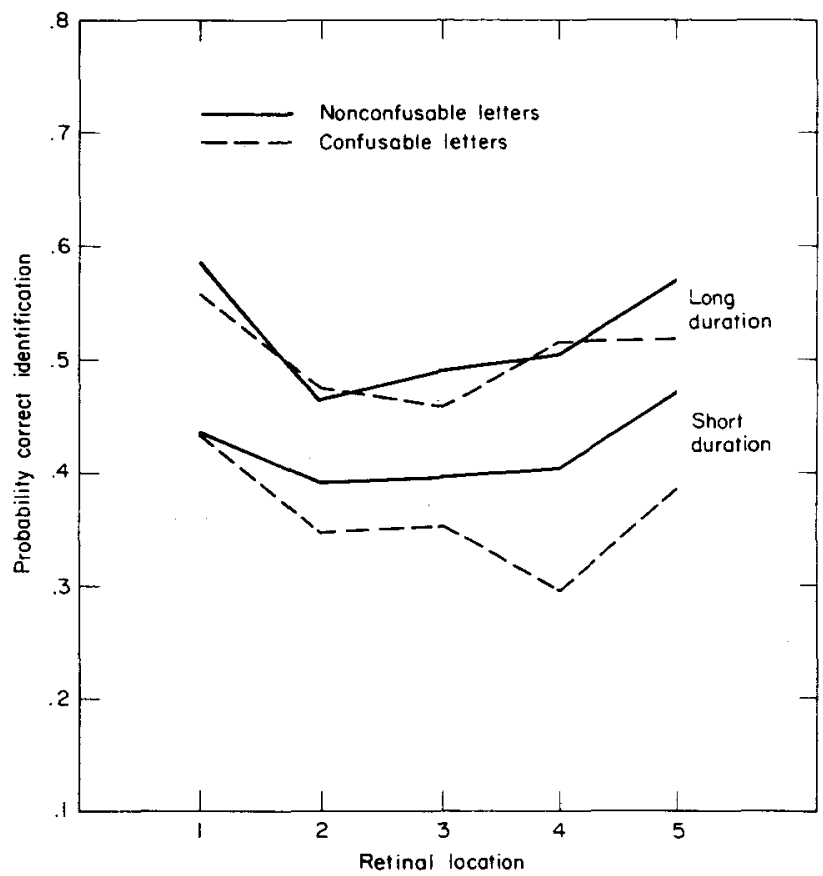

Figure 2. Probability of correct letter identification as a function of retinal location, duration, and level of confusability, collapsed across visual fields.

comparison can be made at Retinal Location 3 as a function of relative position. At this retinal location, the difference between performance on nonconfusable and confusable trials was smaller for central letters (.029) than for peripheral letters (.047), although this difference was not significant. The effect of level of confusability was independent of interletter distance. The average difference beween the nonconfusable and confusable conditions was .036 for trials on which the letters were close (Interletter Distance 1) and .040 for trials on which the letters were spaced (Interletter Distance 2).

In order to determine whether omission errors, incorrect identifications, or both types of errors accounted for the difference in performance between the nonconfusable and confusable conditions, each trial was scored according to how many letter

Table 2

Difference Between Performance on Nonconfusable and Confusable Trials as a Function of Relative Position and Interletter Distance

\begin{tabular}{cccc}
\hline Retinal Location & \multicolumn{3}{c}{ Ketinal Location } \\
\hline Relative Position: Central & \multicolumn{2}{c}{ Relative Position: Peripheral } \\
1 & .012 & 3 & .047 \\
2 & .019 & 4 & .049 \\
3 & .029 & 5 & .071 \\
Interletter Distance: & Interletter Distance: 2 \\
2 & .019 & 1 & .012 \\
3 & .039 & 3 & .037 \\
4 & .049 & 5 & .071 \\
\hline
\end{tabular}


responses were made on the trial and how many of the letter responses were correct identifications. The proportion of confusable trials on which a no-letter or a one-letter response was made (.227 and .417 , respectively) was larger than the proportion of nonconfusable trials on which a no-letter or a one-letter response was made (.210 and .401 respectively), whereas fewer two-letter responses occurred on confusable than on nonconfusable trials. Thus, more omission errors occurred when confusable letters were presented. On those trials on which a one letter response was made, the probability that the responded letter was correct was lower for confusable trials (.743) than for nonconfusable trials (.776). Similarly, on those trials on which a two-letter response was made, the probability that neither responded letter was correct was higher for confusable (.087) than for nonconfusable (.054) letters, the probability that one letter was correct was higher for nonconfusable (.320) than for confusable (.309) letters, and the probability that both letters were correct was higher for nonconfusable (.627) than for confusable (.604) letters. Thus, in addition to a larger number of omission errors, more incorrect identifications occurred when confusable letters were presented.

Chi-squared tests were performed to see whether correctly identifying the target was independent of correctly identifying the distractor. A Fisher-Pearson test (Gordon, Loveland, \& Cureton, 1952) yielded a $\chi^{2}(32)=90.45, \mathrm{p}<.001$, with the probability of identifying both letters larger than the product of the probabilities of identifying the target and the distractor.

\section{Inversion Errors as a Function of Level of Confusability, Number of Correct Identifications, Interletter Distance, and Duration}

Since subjects were instructed to make their responses in a left-to-right order when two identifications were being made, the probability of order inversion can be determined for those trials on which subjects made two-letter responses with at least one correct identification. On these trials, there was a somewhat greater probability of order inversion if the letters were confusable than if they were nonconfusable. A Fisher-Pearson test on the data from the 12 subjects having large enough cell frequencies yielded a $\chi^{2}(24)=31.74, .10<\mathrm{p}<.20$. The probability of order inversion was highly correlated with the number (one or two) of correct identifications on the trial $\left[\chi^{2}(11)=149.80, \mathrm{p}<.001\right]$, with more order inversions occurring when only one letter identification was correct. This value is based on 11 subjects with large enough cell frequencies to compute chi-square values. The probability of order inversion on those trials allowing location scoring was .159 for Interletter Distance 1 and .158 for Interletter Distance 2. Thus, order inversions were in- dependent of interletter distance. Finally, on trials with one correct identification, 9 subjects showed proportionately more inversion errors at the long duration, while 5 showed proportionately more inversion errors at the short duration. On trials with two correct identifications, 10 subjects showed more inversion errors at the short duration, and 4 showed more inversion errors at the long duration. The data from two subjects yielded no information about location performance as a function of duration, since very few responses consisted of two letters. Thus, the results indicate that the probability of order inversion was largely independent of stimulus duration.

\section{DISCUSSION}

In the present experiment, identification performance in a report paradigm was better if the letters were nonconfusable in terms of visual features than if the letters were confusable. This result is consistent with the results of various detection experiments (Estes, 1972; Gardner, 1973; McIntyre, Fox, \& Neale, 1970; Shiffrin \& Gardner, 1972). The interactive channels model (Estes, 1972, 1974) would account for this effect in terms of inhibition occurring between input channels going to the same feature detector when confusable letters, which share features, are presented. This result, however, is opposite to that predicted by the feature perturbation model (Wolford, 1975). According to this model, the arrival in a feature group of a feature common to the letters would generally not interfere with, and may possibly facilitate, the identification process. This is similar to the argument made by Gilmore (1975). Since confusable letters have more common features than nonconfusable letters, the result of perturbations between feature groups would generally seem to be to enhance performance for confusable letters relative to nonconfusable letters, contrary to the present result.

Under the stimulus conditions used in the present experiment, no interaction was found between level of confusability and interletter distance. Both the interactive channels model (Estes, 1972, 1974) and the feature perturbation model (Wolford, 1975) would, however, predict an interaction. In the former, the strength of inhibition is assumed to decrease as interletter distance increases, and in the latter, the probability of feature perturbation is assumed to be a function of interletter distance and, since inhibition and feature perturbations are assumed to have differential effects for confusable and nonconfusable letters, an interaction would be expected. It is possible, though, that such an interaction would be found if a wider range of interletter distances or more variation in interletter confusability were used than in the present experiment.

Although overall performance did not decrease 
significantly with increased distance from the fixation point, the difference between the nonconfusable and confusable conditions did increase with this distance. This is consistent with the interactive channels model (Estes, 1972, 1974), in which it is assumed that competition for access to feature detectors increases at peripheral locations due to a decrease in the number of input channels at peripheral locations. It is difficult to know whether or not the feature perturbation model (Wolford, 1975) would predict this interaction, since in this model it is assumed that with increased distance into the periphery fewer features are extracted and more permutations occur, and the combined effect of these variable on confusable and nonconfusable letters is hard to assess.

A large effect of relative position was found with higher identification accuracy at a position if the second letter occupied a more central, rather than a more peripheral, retinal location. A similar effect was noted in Bouma (1970) and was termed the "discontinuity effect" in the study of Estes et al. (1976). It is also similar to the facilitating effect of a blank space inserted on the peripheral side of a letter location relative to a blank inserted on the foveal side (Estes \& Wolford, 1971; Shaw, 1969; Wolford \& Hollingsworth, 1974a). In terms of the feature perturbation model (Wolford, 1975), this effect could be accounted for by the assumed tendency for detrimental feature transpositions to occur in a foveal, rather than in a peripheral, direction. If it is assumed that transposition errors reflect feature transpositions, this assumption might also be considered consistent with the finding that there is a tendency for incorrectly located letters to move foveally from the correct location in the subject's response (Estes et al., 1976; Krumhansl \& Thomas, 1976).

In addition, the difference between the nonconfusable and the confusable conditions tended to be smaller on the more central letter than on the more peripheral letter. Since retinal location is confounded with relative position, no statistical test can be made for this interaction based on the data from all retinal locations. The pattern, however, was fairly consistent across retinal location, interletter distance, and duration. This effect might be considered consistent with the feature perturbation model (Wolford, 1975 ) if it is assumed that perturbations, which tend to occur in a foveal direction, are more detrimental when the letters are less confusable. This would be true because, for nonconfusable letters which share fewer common features, there would be a greater probability that an incoming feature would be incompatible with the other features in the group. The net effect would be to reduce the difference between the nonconfusable and the confusable conditions at the central position relative to the peripheral position, consistent with the present result. The interactive channels model makes no predictions concerning relative position or an interaction between relative position and level of confusability.

The overall effect of increasing duration was an increase in identification performance, consistent with other studies (see, for example, Estes et al., 1976). This would be accounted for by both the interactive channels model (Estes, 1972, 1974) and the feature perturbation model (Wolford, 1975) in terms of a greater number of features being extracted at longer durations. An interaction between level of confusability and duration was also found in the present experiment such that there was a smaller effect of level of confusability at the longer duration. Although the interactive channels model (Estes, 1972, 1974) makes no direct prediction as to how level of confusability would be expected to interact with duration, the result could be accommodated within the framework of this model if it were assumed that the strength of inhibition is large at short durations but decreases at longer durations. The feature perturbation model (Wolford, 1975) might also predict that increasing duration would have a larger facilitating effect for confusable letters than for nonconfusable letters, since an increase in the number of features might mean an increase in the number of perturbations, which are particularly detrimental in the case of nonconfusable letters, which have fewer features in common.

A large correlation was found in the present experiment between identification performance on one letter and identification performance on the second letter, such that the probability of correctly identifying both letters is larger than the product of the probabilities of correctly identifying each individual letter. The feature perturbation model (Wolford, 1975) offers an explanation for this correlation which does not depend on the notion of correlated sensitivity or attention, since good performance at one location indicates that few feature transpositions have occurred, and thus identification of the second letter would also be expected to be fairly accurate. The interactive channels model (Estes, 1972, 1974), however, might predict negative correlations in the absence of attentional effects, since, if more features are extracted from one location, there is a higher probability that these features inhibit the extraction of similar features from the second location.

Finally, whether or not the subject has made an inversion error in the response can be determined in the present experiment on those trials on which the subject responds with two letters at least one of which is a correct identification. On these trials, there was a correlation between whether or not the letters were in the correct order and the number of 
correct identifications, with correct ordering occurring more often on trials on which two, rather than one, correct identifications were made. Under the assumption that order inversions reflect feature transpositions, this result is consistent with the notion that feature transpositions are detrimental to the identification process. There was a greater probability of order inversion if the letters were confusable rather than nonconfusable. If order inversions result when the distinct features of the peripheral letter are contained in the central feature group and the distinct features of the central letter are contained in the peripheral feature group, then more transpositions might be expected for confusable letters. This would be true since confusable letters have fewer distinct features, and, hence, an order inversion could result when a smaller number of feature transpositions have occurred.

The probability of correct order of report was not correlated in the present experiment with the duration of presentation. This is consistent with the result of the Estes et al. (1976) study, in which the probability of correctly locating a letter in the response did not increase markedly with increased display duration when fixation was controlled. Again, if it is assumed that order inversions reflect feature transpositions, then this result would indicate that the probability of feature transposition is not a function of stimulus duration. In a number of studies, typical U-shaped serial position curves are generated even at extended viewing times (Estes et al., 1976; Taylor \& Brown, 1972; Townsend, Taylor, \& Brown, 1971). In the present experiment, the effect of relative position in the display (whether central or peripheral) was fairly independent of duration. If it is assumed, as in the feature perturbation model (Wolford, 1975), that feature transpositions are responsible for these serial position effects, then these studies are further support for the notion that the probability of feature transposition is not a function of display duration.

In summary, the results of the present experiment lend support for aspects of both the interactive channels (Estes, 1972, 1974) and the feature perturbation (Wolford, 1975) models. The results concerning the overall effect of level of confusability and its interaction with retinal location support the notion of interference, which increases with increased distance from the center of the visual field, between identical or similar features when confusable letters are presented. The results concerning relative position and its interaction with level of confusability support the notion of feature transpositions, with foveal transpositions more likely than peripheral transpositions, and transpositions more detrimental when nonconfusable rather than confusable letters are presented. These results, then, suggest that it might be useful to incorporate both of these notions into a single model. Hopefully, such an approach would allow us to assess the relative importance of these two mechanisms in determining performance.

\section{THE MODEL}

Although the model is proposed to describe the simple situation in which two letters are presented on a trial for identification, it could be extended to describe identification performance when longer letter strings are presented. The central assumptions of the model are:

(1) Information in the form of letter features is extracted from each of the two letter locations.

(2) Of these features, there are identical or very similar features common to the two letter locations, and inhibition or some other form of interference may occur between similar features or duplicate instances of the same feature.

(3) Features may move between the feature groups, each group corresponding to a letter location.

(4) Identification is based on the features in each group after perturbations and interference have occurred, such that the probability of correct identification is proportional to the number of features contained in a feature group, as long as these features are mutually compatible, that is, belong to the feature set of some letter. Very similar or identical features common to the two letter locations are assumed to be compatible with the features from either letter. If the features contained in a feature group are not mutually compatible, then the probability of correct identification is zero.

The first and fourth assumptions are common to a number of models in which it is assumed that letter identifications are based on feature information extracted from letter displays (Estes, 1972; Rumelhart, 1970; Selfridge \& Neisser, 1960; Shiffrin \& Geisler, 1973). The proportional relationship between the probability of letter identification and the number of mutually compatible features contained in a feature set is chosen for simplicity and reflects the notion that the more feature information available, the greater the probability that the features contained in a feature group uniquely determines the identity of the letter from which the features were extracted. This assumption varies somewhat from the corresponding assumption made in the feature perturbation model (Wolford, 1975), which assumes a particular feature set for letters. The assumption concerning the effect on identification of incompatible features in the present model is, however, identical to that made in Wolford's (1975) model. The second assumption concerning inhibition or some other form of interference between similar or identical features shared by the presented letters 
is drawn from the interactive channels model (Estes, 1972 , 1974). And the third assumption concerning feature perturbations is adapted from the feature perturbation model (Wolford, 1975) for the simple situation considered here of letter strings of Length 2 .

Expressions for the probability of correct letter identifications for the two relative positions in the string are given in terms of the following parameters. Let: $\mathbf{N}=$ total number of compativle features required in a feature group for perfect identification; $\mathbf{n}=$ number of features extracted from each letter location on a trial; $\mathbf{k}=$ number of similar or identical features shared by the two letter locations; $a_{F}=$ probability of a foveal feature transposition; $a_{p}=$ probability of a peripheral feature transposition; $\alpha=$ probability that, after possible interference and transposition, one instance of a common feature is contained in the foveal feature group; and $\beta=$ probability that, after possible interference and transposition, one instance of a common feature is contained in the peripheral feature group. The probability of correctly identifying the more central letter on a trial is given by:

$$
\begin{aligned}
P\left(L_{1}\right)= & \frac{b_{F}{ }^{n-k}}{N}\left[(n-k) b_{P}+k \alpha\right] \\
& +\frac{a_{F}^{n-k}}{N}\left[(n-k) a_{P}+k \beta\right]
\end{aligned}
$$

where $b_{F}=1-a_{F}, b_{P}=1-a_{P}$. The probability of correctly identifying the more peripheral letter is given by:

$$
\begin{aligned}
P\left(L_{2}\right)= & \frac{b_{P}{ }^{n-k}}{N}\left[(n-k) b_{F}+k \beta\right] \\
& +\frac{a_{P}{ }^{n-k}}{N}\left[(n-k) a_{F}+k \alpha\right] .
\end{aligned}
$$

The derivation of Equation 1 is given in the Appendix; Equation 2 is derived similarly. In incorporating aspects of both the interactive channels model (Estes, 1972, 1974) and the feature perturbation model (Wolford, 1975), it is hoped that the present model will be able to deal with a wider range of effects than either model alone. In addition, the present model has several advantages over the other two models. The model of Estes $(1972,1974)$ is not quantitatively formulated so that predictions concerning the magnitude of such effects as level of confusability, retinal location, and their interaction cannot be derived directly from the model. On the other hand, while the model of Wolford (1975) can account quantitatively for various effects, the model takes the form of a simulation. The cost of working with such a model is that it is difficult to determine the relative importance of the various assumptions in explaining such effects as retinal location and serial position. The present model is able to overcome these limitations, since analytic expressions for identification performance in terms of parameters describing such mechanisms as interference and transpositions can be derived.

In using the data from the present experiment to estimate the values of the parameters of the model, certain assumptions were made in the interest of keeping the number of parameters fairly small. Under the assumption that order inversion errors reflect feature transpositions, the probabilities of feature transpositions, $a_{F}$ and $a_{P}$, were assumed to be independent of duration, since duration was not found to affect the probability of order inversions. The probability of feature transposition was, however, allowed to vary with the direction of movement (whether peripheral or foveal), interletter distance, and retinal location. Since there was no linear trend found in identification performance with increased distance from the center of the visual field, the number, $n$, of features extracted from each retinal location was assumed constant, although $n$ was allowed to vary with duration.

In the model, the effect of level of confusability is accounted for in terms of the parameters $k$, the number of similar or identical features common to the two locations, and $\alpha$ and $\beta$, the probabilities that a common feature is contained in the two feature groups after possible interference and perturbations have occurred. The sum of $\alpha$ and $\beta$, which equals the average number $(\leqslant 2)$ of occurrences of a common feature in the pair of feature groups, can be considered a measure of the strength of inhibition between the two similar features, since, as the strength of inhibition increases, this average number of occurrences would be expected to decrease. The possibility that confusability affects two sets of variables, $\mathrm{k}$ and $(\alpha, \beta)$, and the possibility of interpreting $(\alpha, \beta)$ in two ways, as an index of confusability and of inhibition, limits the ability of the model to uniquely associate construct with parameter. Indeed, this conclusion was confirmed empirically by our finding that the data are about equally well accounted for by estimation procedures that fix $k$ and allow $(\alpha, \beta)$ to vary with stimulus type or by procedures that fix $(\alpha, \beta)$ and vary $\mathrm{k}$. We will report only the results of one of the latter set of procedures.

To derive the estimates shown below, we assumed that $\alpha$ was independent of stimulus type and that $\alpha+\beta=1$. Also, in the interest of keeping the number of parameters small, it was assumed that the value of $k$, the number of common features, was zero for letters termed nonconfusable in the present 
study, and that for confusable letters the values of $k$ for the different stimulus configurations at the long duration were proportional, with proportionality constant $p$, to the values of $k$ for the different configurations at the short duration.

Table 3 shows the parameter estimates based on the 32 data points corresponding to the two levels of confusability, two durations, and two relative positions for the four possible stimulus configurations (Retinal Locations 1 and 3,2 and 3, 3 and 4, or 3 and 5). The value of the loss function, the sum of squared deviations, is given in Table 3 , and the fit of the model to the 32 data points is shown in Figure 3.

The estimated parameter values indicate that the mean number of features extracted from each letter location increased with duration [n(short) $=2.50$, $\mathrm{n}$ (long) $=3.18$ ], and the mean number of features required for perfect identification, $\mathrm{N}$, is estimated as 5.03. The mean number of similar or identical features common to the two locations when confusable letters were presented showed a general increase with increased average retinal location and was generally higher when the letters were presented at Interletter Distance 1 than at Interletter Distance 2. The proportionality constant for the effect on $k$ of duration, $p$, was estimated as .31 , indicating that at the longer duration there was less uncertainty about feature identity, reducing the number of features from one letter group that appear similar to features in the other letter group. Given our assumption that exactly one instance of a duplicate pair is lost, we found that the instance is deleted equally often from the two letter groups $(\alpha=.47 \sim .5)$. Finally, the estimates of $\mathrm{a}_{\mathrm{F}}$ indicated an increasing probability of foveal transposition with increased average retinal location and with decreased interletter distance. The estimated values of ap, the probability of peripheral transposition, were relatively small, and generally decreased with distance into the periphery, again indicating an increasingly strong bias toward foveal movement with increased distance from the center of the visual field.

Because we set $\mathrm{k}=0$ for nonconfusable letterpairs and fixed $\alpha$ and $\beta$, the observed variation in the estimates of $\mathbf{k}$ across stimulus types could be due to variation in the effects of mutual inhibition between similar or identical features from confusable letter-

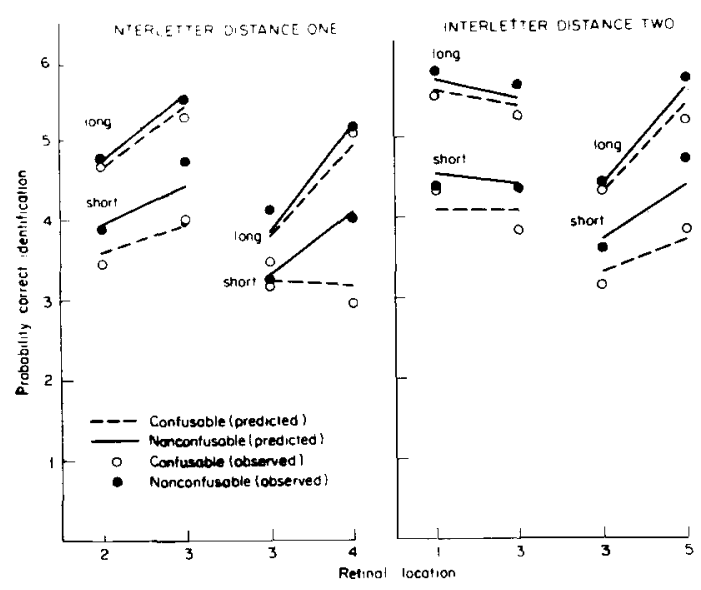

Figure 3. Predicted and observed probability of correct letter identification as a function of interletter distance, retinal location, duration, relative position, and level of confusability, collapsed across visual fields.

pairs. Therefore, $\mathrm{k}$ measures not only the physical or objective similarity between letters, but also the effects of inhibition on the perception of similar feature pairs. In other words, $k$ is properly regarded as a measure of the subjective or effective similarity (that is, after inhibition) between two letters. Our estimates of $k$ and of $a_{F}$ and $a_{P}$ suggest that the decline in performance on confusable pairs, as average distance from the fixation point increases and as interletter distance decreases, is due both to an increase in effective similarity between the letters and to an increase in the probability of (foveal) feature perturbations. Other estimation procedures, in which $k$ is fixed and $\alpha$ and $\beta$ are allowed to vary, lead to the same conclusion (here "effective similarity" is high when $\alpha$ and $\beta$ are low).

\section{CONCLUSION}

The present experiment was designed to investigate the effect of level of confusability between presented characters on identification performance, and the interaction between level of confusability and such display variables as retinal location, interletter distance, relative location, and stimulus duration. The results of the present study support the notion that interference between similar or identical features may occur when confusable letters are presented,

Table 3

Estimated Parameter Values

\begin{tabular}{|c|c|c|c|}
\hline $\mathrm{n}(\operatorname{sh}(\mathrm{r}) \mathrm{t})=2.50$ & $\mathrm{n}$ (long) $=3.18$ & $\mathrm{~N}=5.03$ & \\
\hline$k(1,3)=.53$ & $k(2,3)=.69$ & $k(3,4)=1.78$ & $k(3,5)=1.03$ \\
\hline$p=.31$ & $\alpha=.47$ & & \\
\hline$a_{F}(1,3)=.018$ & $a_{F}(2,3)=.084$ & $a_{F}(3,4)=.147$ & $a_{F}(3,5)=.111$ \\
\hline$\left.a_{p}(1.3)=.041\right)$ & $a_{p}(2,3)=.012$ & $a_{p}(3,4)=.009$ & $a_{p}(3,5)=.000$ \\
\hline
\end{tabular}

Note l.oss function $=.0106$ 
as suggested in the interactive channels model (Estes, 1972, 1974). In addition, support comes from the present experiment for the notion that extracted features may be incorrectly assigned to feature groups with a detrimental effect on identification performance, as suggested by the feature perturbation model (Wolford, 1975). A model incorporating aspects of both these models is proposed to account for the results of the present study, and leads to interpretable estimates of the parameters describing interference and feature perturbations.

\section{APPENDIX}

The derivation of Equation 1, which gives the probability of correctly identifying the central leter, will be shown. For any trial on which the $\mathrm{n}-\mathrm{k}$ distinct features from the peripheral letter are divided between the two feature groups, the probability of identifying the central letter is zero. So only two cases need to be considered: (i) the case in which all distinct features from the peripheral letter are contained in Feature Group 2 (with probability $\mathrm{b}_{\mathrm{F}}{ }^{\mathrm{n}-\mathrm{k}}$ ), and (ii) the case in which all distinct features from the peripheral letter are contained in Feature Group 1 (with probability $a F^{n-k}$ ). First consider Case $i$, in which all of the features from the peripheral letter are contained in Feature Group 2. In this case, a correct identification of the central letter can only be made based on the features in Feature Group 1. First, consider the $k$ common feature pairs. The probabilty that an instance of a common pair is contained in Feature Group 1 is $\alpha$. Of the $k$ features, there are ( $\left.\begin{array}{c}k \\ i\end{array}\right)$ ways of having $i$ of these features in Feature Group 1 , each with probability $\alpha^{\mathrm{i}}(1-\alpha)^{\mathrm{k}-\mathrm{i}}$. Next, consider the $n-k$ distinct central letter features. There $\operatorname{are}\left({ }^{n-k}\right)$ ways of forming a group of $j$ of these features in Feature Group 1, each with probability of $b_{P} a_{P} a^{n-k-j}$. The probability of identifying the central letter is, then, $(j+i) / N$, where $j$ and $i$ are as defined. In Case $i$ for $a$ given value of $i$, the probability of identifying the central letter is given by:

$$
\begin{gathered}
\left.\sum_{j=0}^{n=k}\left(\frac{j+i}{N}\right) \begin{array}{c}
n-k \\
j
\end{array}\right) b_{P} a_{P} a^{n-k-j} \\
=\frac{1}{N}\left[\sum_{j=0}^{n-k} j(n-k) b_{P} j_{P}{ }^{n-k-j}\right. \\
\left.\quad+i \sum_{j=0}^{n-k}(n-k) b_{P} a_{P} n-k-j\right] \\
=\frac{1}{N}\left[(n-k) b_{P}+i\right],
\end{gathered}
$$

since the first summation in brackets is the expected value of a binomial distribution with parameters bp and $n-k$, and the second summation is the binomial expansion of $\left(b_{P}+a_{P}\right)^{n-k}$ with $b_{P}+a_{P}=1$. So, the total probability of correctly identifying the central letter and being in Case $i$ is:

$$
\begin{aligned}
& \sum_{i=0}^{k}\left(\begin{array}{l}
k \\
i
\end{array}\right) \alpha^{i}(1-\alpha)^{k-i} \frac{b_{F} n-k}{N}\left[(n-k) b_{P}+i\right] \\
& =\frac{b_{F}{ }^{n-k}}{N}\left[(n-k) b_{P} \sum_{i=0}^{k} \quad\left(\begin{array}{l}
k \\
i
\end{array}\right) \alpha^{i}(1-\alpha)^{k-i}\right. \\
& \left.+\sum_{i=0}^{k} \mathrm{i}\left(\begin{array}{l}
\mathrm{k} \\
\mathrm{i}
\end{array}\right) \alpha^{\mathrm{i}}(1-\alpha)^{\mathrm{k}-\mathrm{i}}\right] \\
& =\frac{b_{F}{ }^{n-k}}{N} \times\left[(n-k) b_{P}+k \alpha\right] \text {. }
\end{aligned}
$$

Similarly, the probability of being in Case ii and correctly identifying the central letter is given by

$$
\frac{a_{F}^{n-k}}{N}\left[(n-k) a_{P}+k \beta\right],
$$

where $\beta$ is the probability that an instance of a redundant feature pair is contained in Feature Group 2. So the total probability of identifying the central letter is:

$$
\begin{aligned}
P\left(L_{1}\right)= & \frac{b_{F}^{n-k}}{N}\left[(n-k) b_{P}+k \alpha\right] \\
& +\frac{a_{F}^{n-k}}{N}\left[(n-k) a_{P}+k \beta\right] .
\end{aligned}
$$

\section{REFERENCES}

Bouma, H. Interaction effects in parafoveal letter recognition. Nature, 1970, 226, 177-178.

Bryden, M. P. Accuracy and order of report in tachistoscopic recognition. Canadian Journal of Psychology, 1966, 20, 262-272.

Crovitz, H. F., \& Schiffman, H. R. Visual field and the letter span. Journal of Experimental Psychology, 1965, 70, 218-223.

Eriksen, B. A., \& Eriksen, C. W. Effects of noise letters upon the identification of a target letter in a nonsearch task. Perception \& Psychophysics, 1974, 16, 143-149.

EsTES, W. K. Interactions of signal and background variables in visual processing. Perception \& Psychophysics, 1972, 12, 278-286.

EsTES, W. K. Redundancy of noise elements and signals in visual detection of letters. Perception \& Psychophysics, 1974, 16, 53-60.

Estes, W. K., Allmeyer, D. H., \& Reder, S. M. Serial position functions for letter identification at brief and extended exposure durations. Perception \& Psychophysics, 1976, 19, 1-15.

Estes, W. K., \& Wolford, G. L. Effects of spaces on report from tachistoscopically presented letter strings. Psychonomic Science, $1971,25,77-80$.

GARDNER, G. T. Evidence for independent parallel channels in tachistoscopic perception. Cognitive Psychology, 1973, 4, 130-155.

GrLmore, G. C. The interaction among letters in a visual display. Unpublished doctoral dissertation, The Johns Hopkins University, 1975.

Gordon, M. H., Loveland, E. H., \& Cureton, E. E. An extended table of chi-square for two degrees of freedom, for use in combining probabilities from independent samples. Psychometrika, 1952, 17, 311-316.

Krumhanse, C. L., \& Thomas, E. A. C. Extracting identity and 
location information from briefly presented letter arrays. Perception \& Psychophysics. 1976. 20. 243-258.

McIntyre, C., Fox, R., \& Neale, J. Effects of noise similarity and redundancy on the information processed from brief visual displays. Perception \& Psychophysics, 1970, 7, 328-332.

RUMELhart, D. E. A multicomponent theory of the perception of briefly exposed visual displays. Journal of Mathematical Psychology. 1970, 7, 191-218.

Selfridge, O. G., \& Neisser. U. Pattern recognition by machine. Scientific American, 1960, 203, 60-68.

SHAW, P. Processing of tachistoscopic displays with controlled order of characters and spaces. Perception \& Psychophysics, $1969,6,257-266$

ShIFFrin, R. M. \& G GARDNER, G. T. Visual processing capacity and attentional control. Journal of Experimental Psychology, 1972, 93, 72-82.

SHIfFRIN. R. M.. \& GeISLER, W. S. Visual recognition in a theory of information processing. In R. L. Solso (Ed.), Contemporary issues in cognitive psychology: The Lovola symposium. Washington, D.C: Winston, 1973.

Strangert, B., \& BränNSTröm, L. Spatial interaction effects in letter processing. Perception \& Psychophysics, 1975, 17, 268-272.

TAYLOR, S. G., \& Brown, D. R. Lateral visual masking: Supraretinal effects when viewing linear arrays with unlimited viewing time. Perception \& Psychophysics. 1972, 12. 97.99.
Townsend, J. T. Theoretical analysis of an alphabetic confusion matrix. Perception \& Psychophysics, 1971, 9. 40-50.

Townsend, J. T.. Taylor, S. G.. \& Brown, D. R. Lateral masking for letters with unlimited viewing time. Perception \& Psychophysics, 1971, 10, 375-378.

Wol.ford, G. Perturbation model for letter identification. Psychological Review, 1975, 82, 184-199.

WOLFORD, G., \& HOLLINGSWORTH, S. Lateral masking in visual information processing. Perception \& Psychophysics. 1974. 16. 315-320. (a)

WOLFORD, G., \& Hollingsworth. S. Retinal location and string position as important variables in visual information processing. Perception \& Psychophysics, 1974, 16, 437-442. (b)

\section{NOTE}

1. The effect on letter identification of the direction (whether peripheral or foveal) of a second, simultaneously presented letter could, however, be offset by the decreased probability of feature extraction at the more peripheral location than at the more central location.

(Received for publication August 30, 1976; revision accepted December 6, 1976.) 